Article

\title{
Positive Solutions for a Class of $p$-Laplacian Hadamard Fractional-Order Three-Point Boundary Value Problems
}

\author{
Jiafa $\mathrm{Xu}^{1}{ }^{1}$, Jiqiang Jiang ${ }^{2, *}$ and Donal $\mathrm{O}^{\prime}$ Regan $^{3}$ \\ 1 School of Mathematical Sciences, Chongqing Normal University, Chongqing 401331, China; \\ 20150028@cqnu.edu.cn \\ 2 School of Mathematical Sciences, Qufu Normal University, Qufu 273165, China \\ 3 School of Mathematics, Statistics and Applied Mathematics, National University of Ireland, \\ H91 TK33 Galway, Ireland; donal.oregan@nuigalway.ie \\ * Correspondence: qfjjq@qfnu.edu.cn
}

Received: 3 February 2020; Accepted: 25 February 2020; Published: 27 February 2020

check for updates

\begin{abstract}
In this paper, using the Avery-Henderson fixed point theorem and the monotone iterative technique, we investigate the existence of positive solutions for a class of $p$-Laplacian Hadamard fractional-order three-point boundary value problems.
\end{abstract}

Keywords: Hadamard fractional boundary value problem; Avery-Henderson fixed point theorem; monotone iterative technique; positive solutions

\section{Introduction}

In this paper, we study the following $p$-Laplacian Hadamard fractional-order three-point boundary value problem

$$
\left\{\begin{array}{l}
-D^{\alpha}\left(\varphi_{p}\left(D^{\beta} y(t)\right)\right)=f(t, y(t)), t \in(1, e), \\
y(1)=y(e)=\delta y(1)=\delta y(e)=0, D^{\beta} y(1)=0, D^{\beta} y(e)=b D^{\beta} y(\eta),
\end{array}\right.
$$

where $\alpha \in(1,2], \beta \in(3,4]$ are real numbers, and $D^{\alpha}, D^{\beta}$ are the Hadamard fractional derivatives; $\delta$ means that delta derivative, i.e., $\delta y(1)=\left.\left(t \frac{d y}{d t}\right)\right|_{t=1^{\prime}} \delta y(e)=\left.\left(t \frac{d y}{d t}\right)\right|_{t=e^{\prime}} \varphi_{p}$ is the $p$-Laplacian, i.e., $\varphi_{p}(s)=|s|^{p-2} s$ with $s \in \mathbb{R}, p>1$. The constants $b, \eta$ and the function $f$ satisfy the conditions:

(H1) $b \in[0,+\infty)$ and $\eta \in(1, e)$ with $b^{p-1}(\log \eta)^{\alpha-1} \in[0,1)$.

(H2) $f \in C\left([1, e] \times \mathbb{R}^{+}, \mathbb{R}^{+}\right)$.

Fractional calculus arises naturally in describing complex phenomena in many applications. For example, Podlubny [1] introduces a fractional electric circuit model, and gives a closed-loop control system

$$
\left\{\begin{array}{l}
{ }_{0} D_{t}^{q} x(t)=\alpha_{0} D_{t}^{q-1}(y(t)-x(t))-\frac{2 \alpha}{7}\left(4 x(t)-x^{3}(t)\right) \\
\frac{\mathrm{d} y(t)}{\mathrm{d} t}=x(t)-y(t)+z(t) \\
\frac{\mathrm{d} z(t)}{\mathrm{d} t}=-\frac{100}{7} y(t)
\end{array}\right.
$$


where ${ }_{0} D_{t}^{q},{ }_{0} D_{t}^{q-1}$ are fractional derivatives. For more details on fractional applications, we refer the reader to [2-18]. In [6], K. Diethelm and N. J. Ford studied numerical solutions for fractional differential equations of the form

$$
y^{(\alpha)}(t)=f\left(t, y(t), y^{\left(\beta_{1}\right)}(t), y^{\left(\beta_{2}\right)}(t), \ldots, y^{\left(\beta_{n}\right)}(t)\right),
$$

with $\alpha>\beta_{n}>\beta_{n-1}>\cdots>\beta_{1}$ and $\alpha-\beta_{n} \leq 1, \beta_{j}-\beta_{j-1} \leq 1,0<\beta_{1} \leq 1$. They not only discussed the analytical question on solutions, but also investigated how the solutions depend on the given data. In [7], Y. Luchko studied the generalized time-fractional diffusion equation

$$
D_{t}^{\alpha} u(t)=\operatorname{div}(p(x) \operatorname{grad} u)-q(x) u+F(x, t),
$$

where $0<\alpha \leq 1,(x, t) \in G \times(0, T), G \subset \mathbb{R}^{n}$, and $D_{t}^{\alpha}$ is the Caputo-Dzherbashyan fractional derivative. The author used an appropriate maximum principle to obtain a unique solution, and also studied the continuous dependence on the data given in the problem.

Research on Hadamard fractional differential equations is at an early stage; see for example [19-30]. In [19], B. Ahmad and S. K. Ntouyas used fixed point theory to study the existence and uniqueness of solutions for a Hadamard type fractional differential equation involving integral boundary conditions

$$
\left\{\begin{array}{l}
D^{\alpha} x(t)=f(t, x(t)), 1<t<e, 1<\alpha \leq 2, \\
x(1)=0, \quad x(e)=\frac{1}{\Gamma(\beta)} \int_{1}^{e}\left(\log \frac{e}{s}\right)^{\beta-1} \frac{x(s)}{s} \mathrm{~d} s, \beta>0,
\end{array}\right.
$$

where $f$ satisfies the Lipschitz condition.

On the other hand, $p$-Laplacian equations are extensively used in physics, mechanics, dynamical systems, etc (see [15-18,20-23,31]). For example, Leibenson [31] introduced $p$-Laplacian differential equations to study a mechanics problem involving turbulent flow in a porous medium. Recently, G. Wang et al. used the tools of Hadamard type fractional-order equations to study turbulent flow models, see $[20,21]$. In [20], they studied the uniqueness, the existence and nonexistence of solutions for the following Hadamard type fractional differential equation with the $p$-Laplacian operator

$$
\left\{\begin{array}{l}
-D^{\beta} \varphi_{p}\left(-D^{\alpha} \chi(t)\right)=f(t, \chi(t), \chi(\theta(t))), \\
D^{\alpha} \chi(1)=D^{\alpha} \chi(e)=0, \chi(1)=0 \\
D^{\alpha-1} \chi(1)=\eta D^{\alpha-1} \chi(e)
\end{array}\right.
$$

where $f \in C\left([1, e] \times \mathbb{R}^{2}, \mathbb{R}\right)$ and $\theta \in C[1, e]$. In [21], they also studied the unique positive solution for a Caputo-Hadamard-type fractional turbulent flow model

$$
\left\{\begin{array}{l}
{ }^{C H} D^{\chi_{2}} \varphi_{p(t)}\left({ }^{C H} D^{\chi_{1}} x(t)\right)+f\left(x(t), I_{\eta}^{\gamma, \delta} x(t)\right)=0, t \in[1, e], \\
x^{\prime}(1)=\lambda x^{\prime}(e), \quad x(1)=x^{\prime \prime}(1)=0,{ }^{C H} D^{\chi_{1}} x(1)=0
\end{array}\right.
$$

where ${ }^{\mathrm{CH}} \mathrm{D}$ is Caputo-Hadamard fractional derivative, $I_{\eta}^{\gamma, \delta}$ is the generalized Erdelyi-Kober fractional integral operator: $I_{\eta}^{\gamma, \delta} f(t)=\frac{\eta t^{-\eta(\delta+\gamma)}}{\Gamma(\delta)} \int_{0}^{t} \frac{s^{\eta \gamma+\eta-1} f(s)}{\left(t^{\eta}-s^{\eta}\right)^{1-\delta}} d s$.

In this paper, we study positive solutions for the $p$-Laplacian Hadamard fractional-order differential Equation (1). Using the monotone iterative technique we show that (1) has two positive solutions, and we establish iterative formulas for the two solutions. In addition from the Avery-Henderson fixed point theorem, we also obtain that (1) has two positive solutions under some appropriate conditions on the nonlinearity $f$. It is interesting to note that the methods used in this paper can be applied to very general integral equations (and therefore very general differential equations) if 
the kernel has a suitable behavior as described in Section 2. The behavior of the Greens' function of a problem will indicate whether the theory presented in this paper can be used efficiently.

\section{Preliminaries}

In this section, we provide the definition of the Hadamard fractional derivative; for other related detail materials see the book [5].

Definition 1. The Hadamard derivative of fractional order $q$ for a function $g:[1, \infty) \rightarrow \mathbb{R}$ is defined as

$$
D^{q} g(t)=\frac{1}{\Gamma(n-q)}\left(t \frac{d}{d t}\right)^{n} \int_{1}^{t}(\log t-\log s)^{n-q-1} g(s) \frac{d s}{s}, n-1<q<n,
$$

where $n=[q]+1,[q]$ denotes the integer part of the real number $q$ and $\log (\cdot)=\log _{e}(\cdot)$.

In (1), we let $\varphi_{p}\left(D^{\beta} y(t)\right)=v(t), t \in[1, e]$. Then we have

$$
\begin{aligned}
& v(1)=\left.\varphi_{p}\left(D^{\beta} y(t)\right)\right|_{t=1}=\left|D^{\beta} y(1)\right|^{p-2} D^{\beta} y(1)=0, \\
& v(e)=\left|D^{\beta} y(e)\right|^{p-2} D^{\beta} y(e)=\left|b D^{\beta} y(\eta)\right|^{p-2} b D^{\beta} y(\eta)=b^{p-1} v(\eta) .
\end{aligned}
$$

Therefore, we obtain

$$
\left\{\begin{array}{l}
-D^{\alpha} v(t)=f(t, y(t)), t \in(1, e) \\
v(1)=0, v(e)=b^{p-1} v(\eta)
\end{array}\right.
$$

where $\alpha \in(1,2]$ and $b, \eta, f$ satisfy (H1)-(H2). Using a similar argument as in Lemmas 2 and 3 of [24], we obtain the following result.

Lemma 1. The boundary value problem (2) is equivalent to the following Hammerstein-type integral equation

$$
v(t)=\int_{1}^{e} H(t, s) f(s, y(s)) d s
$$

where

$$
\begin{aligned}
& H(t, s)=H_{0}(t, s)+\frac{b^{p-1}(\log t)^{\alpha-1}}{1-b^{p-1}(\log \eta)^{\alpha-1}} H_{0}(\eta, s), \text { for } t, s \in[1, e], \\
& H_{0}(t, s)=\frac{1}{\Gamma(\alpha)}\left\{\begin{array}{l}
(\log t)^{\alpha-1}(1-\log s)^{\alpha-1}, 1 \leq t \leq s \leq e, \\
(\log t)^{\alpha-1}(1-\log s)^{\alpha-1}-(\log t-\log s)^{\alpha-1}, 1 \leq s \leq t \leq e .
\end{array}\right.
\end{aligned}
$$

Proof. From the first equation in (2) we have

$$
v(t)=c_{1}(\log t)^{\alpha-1}+c_{2}(\log t)^{\alpha-2}-\frac{1}{\Gamma(\alpha)} \int_{1}^{t}(\log t-\log s)^{\alpha-1} f(s, y(s)) \frac{d s}{s},
$$

where $c_{i} \in \mathbb{R}, i=1,2$ and $v(1)=0$ implies that $c_{2}=0$. Then from $v(e)=b^{p-1} v(\eta)$ we have

$$
c_{1}-\frac{1}{\Gamma(\alpha)} \int_{1}^{e}(1-\log s)^{\alpha-1} f(s, y(s)) \frac{d s}{s}=c_{1} b^{p-1}(\log \eta)^{\alpha-1}-\frac{b^{p-1}}{\Gamma(\alpha)} \int_{1}^{\eta}(\log \eta-\log s)^{\alpha-1} f(s, y(s)) \frac{d s}{s},
$$

and

$$
\begin{aligned}
c_{1}= & \frac{1}{\left(1-b^{p-1}(\log \eta)^{\alpha-1}\right) \Gamma(\alpha)} \int_{1}^{e}(1-\log s)^{\alpha-1} f(s, y(s)) \frac{d s}{s} \\
& -\frac{b^{p-1}}{\left(1-b^{p-1}(\log \eta)^{\alpha-1}\right) \Gamma(\alpha)} \int_{1}^{\eta}(\log \eta-\log s)^{\alpha-1} f(s, y(s)) \frac{d s}{s} .
\end{aligned}
$$

Substituting this $c_{1}$, we obtain 


$$
\begin{aligned}
v(t)= & \frac{1}{\left(1-b^{p-1}(\log \eta)^{\alpha-1}\right) \Gamma(\alpha)} \int_{1}^{e}(\log t)^{\alpha-1}(1-\log s)^{\alpha-1} f(s, y(s)) \frac{d s}{s} \\
& +\frac{1}{\Gamma(\alpha)} \int_{1}^{e}(\log t)^{\alpha-1}(1-\log s)^{\alpha-1} f(s, y(s)) \frac{d s}{s}-\frac{1}{\Gamma(\alpha)} \int_{1}^{e}(\log t)^{\alpha-1}(1-\log s)^{\alpha-1} f(s, y(s)) \frac{d s}{s} \\
& -\frac{1}{\Gamma(\alpha)} \int_{1}^{t}(\log t-\log s)^{\alpha-1} f(s, y(s)) \frac{d s}{s}-\frac{b^{p-1}(\log t)^{\alpha-1}}{\left(1-b^{p-1}(\log \eta)^{\alpha-1}\right) \Gamma(\alpha)} \int_{1}^{\eta}(\log \eta-\log s)^{\alpha-1} f(s, y(s)) \frac{d s}{s} \\
= & \int_{1}^{e} H_{0}(t, s) f(s, y(s)) \frac{d s}{s}+\frac{b^{p-1}(\log t)^{\alpha-1}}{1-b^{p-1}(\log \eta)^{\alpha-1}} \int_{1}^{e} H_{0}(\eta, s) f(s, y(s)) \frac{d s}{s} \\
= & \int_{1}^{e} H(t, s) f(s, y(s)) \frac{d s}{s} .
\end{aligned}
$$

This completes the proof.

Note that

$$
\varphi_{p}\left(D^{\beta} y(t)\right)=v(t)=\int_{1}^{e} H(t, s) f(s, y(s)) \frac{d s}{s}, t \in[1, e] .
$$

Then we have

$$
D^{\beta} y(t)=\varphi_{p}^{-1}\left(\int_{1}^{e} H(t, s) f(s, y(s)) \frac{d s}{s}\right), t \in[1, e]
$$

and together with the boundary conditions $y(1)=y(e)=\delta y(1)=\delta y(e)=0$, we have the following result.

Lemma 2. The problem (3) is equivalent to the following Hammerstein-type integral equation

$$
y(t)=\int_{1}^{e} G(t, s) \varphi_{p}^{-1}\left(\int_{1}^{e} H(s, \tau) f(\tau, y(\tau)) \frac{d \tau}{\tau}\right) \frac{d s}{s}
$$

where

$$
G(t, s)=\left\{\begin{array}{l}
\frac{(\log t-\log s)^{\beta-1}+(\log t)^{\beta-2}(1-\log s)^{\beta-2}[(\log s-\log t)+(\beta-2) \log s(1-\log t)]}{\Gamma(\beta)}, 1 \leq s \leq t \leq e, \\
\frac{(\log t)^{\beta-2}(1-\log s)^{\beta-2}[(\log s-\log t)+(\beta-2) \log s(1-\log t)]}{\Gamma(\beta)}, 1 \leq t \leq s \leq e .
\end{array}\right.
$$

Proof. For convenience, we put $\phi(t)=\varphi_{p}^{-1}\left(\int_{1}^{e} H(t, s) f(s, y(s)) \frac{d s}{s}\right), t \in[1, e]$. Then by a similar argument as in Lemma 1, we have

$$
y(t)=c_{1}(\log t)^{\beta-1}+c_{2}(\log t)^{\beta-2}+c_{3}(\log t)^{\beta-3}+c_{4}(\log t)^{\beta-4}+\frac{1}{\Gamma(\beta)} \int_{1}^{t}(\log t-\log s)^{\beta-1} \phi(s) \frac{d s}{s},
$$

where $c_{i} \in \mathbb{R}, i=1,2,3,4$ and $y(1)=\delta y(1)=0$ implies that $c_{3}=c_{4}=0$. Consequently, we have

$$
\delta y(t)=c_{1}(\beta-1)(\log t)^{\beta-2}+c_{2}(\beta-2)(\log t)^{\beta-3}+\frac{\beta-1}{\Gamma(\beta)} \int_{1}^{t}(\log t-\log s)^{\beta-2} \phi(s) \frac{d s}{s} .
$$

As a result, we have

$$
\begin{aligned}
& c_{1}+c_{2}+\frac{1}{\Gamma(\beta)} \int_{1}^{e}(1-\log s)^{\beta-1} \phi(s) \frac{d s}{s}=0, \\
& c_{1}(\beta-1)+c_{2}(\beta-2)+\frac{\beta-1}{\Gamma(\beta)} \int_{1}^{e}(1-\log s)^{\beta-2} \phi(s) \frac{d s}{s}=0 .
\end{aligned}
$$


Solving this system, we obtain

$$
\left(\begin{array}{l}
c_{1} \\
c_{2}
\end{array}\right)=\left(\begin{array}{cc}
\beta-2 & -1 \\
1-\beta & 1
\end{array}\right)\left(\begin{array}{l}
\frac{1}{\Gamma(\beta)} \int_{1}^{e}(1-\log s)^{\beta-1} \phi(s) \frac{d s}{s} \\
\frac{\beta-1}{\Gamma(\beta)} \int_{1}^{e}(1-\log s)^{\beta-2} \phi(s) \frac{d s}{s}
\end{array}\right) .
$$

Hence, we have

$$
\begin{aligned}
y(t)= & \frac{\beta-2}{\Gamma(\beta)} \int_{1}^{e}(\log t)^{\beta-1}(1-\log s)^{\beta-1} \phi(s) \frac{d s}{s}-\frac{\beta-1}{\Gamma(\beta)} \int_{1}^{e}(\log t)^{\beta-1}(1-\log s)^{\beta-2} \phi(s) \frac{d s}{s} \\
& -\frac{\beta-1}{\Gamma(\beta)} \int_{1}^{e}(\log t)^{\beta-2}(1-\log s)^{\beta-1} \phi(s) \frac{d s}{s}+\frac{\beta-1}{\Gamma(\beta)} \int_{1}^{e}(\log t)^{\beta-2}(1-\log s)^{\beta-2} \phi(s) \frac{d s}{s} \\
& +\frac{1}{\Gamma(\beta)} \int_{1}^{t}(\log t-\log s)^{\beta-1} \phi(s) \frac{d s}{s} \\
= & \frac{1}{\Gamma(\beta)} \int_{1}^{e}(\log t)^{\beta-2}(1-\log s)^{\beta-2}[(\log s-\log t)+(\beta-2) \log s(1-\log t)] \phi(s) \frac{d s}{s} \\
& +\frac{1}{\Gamma(\beta)} \int_{1}^{t}(\log t-\log s)^{\beta-1} \phi(s) \frac{d s}{s} \\
= & \int_{1}^{e} G(t, s) \phi(s) \frac{d s}{s} .
\end{aligned}
$$

Consequently, we find

$$
y(t)=\int_{1}^{e} G(t, s) \varphi_{p}^{-1}\left(\int_{1}^{e} H(s, \tau) f(\tau, y(\tau)) \frac{d \tau}{\tau}\right) \frac{d s}{s} .
$$

This completes the proof.

Lemma 3. The functions $H_{0}$ and $G$ have the following properties:

(i) $H_{0}(t, s) \geq 0, G(t, s) \geq 0$ for $(t, s) \in[1, e] \times[1, e]$,

(ii) $(\beta-2)(\log t)^{\beta-2}(1-\log t)^{2}(\log s)^{2}(1-\log s)^{\beta-2} \leq \Gamma(\beta) G(t, s) \leq M_{0}(\log s)^{2}(1-\log s)^{\beta-2}$ for $(t, s) \in[1, e] \times[1, e]$

(iii) $(\beta-2)(\log s)^{2}(1-\log s)^{\beta-2}(\log t)^{\beta-2}(1-\log t)^{2} \leq \Gamma(\beta) G(t, s) \leq M_{0}(\log t)^{\beta-2}(1-\log t)^{2}$, for $(t, s) \in[1, e] \times[1, e]$, where $M_{0}=\max \left\{\beta-1,(\beta-2)^{2}\right\}$.

Lemma 3 (ii) and (iii) are direct results from Lemma 3 in [14]. Moreover, by Lemma 3 (i) we have $H(t, s) \geq 0$ for $(t, s) \in[1, e] \times[1, e]$.

Let $E:=C[1, e],\|y\|:=\max _{t \in[1, e]}|y(t)|$ and $P:=\{y \in E: y(t) \geq 0, \forall t \in[1, e]\}$. Then $(E,\|\cdot\|)$ is a real Banach space and $P$ a cone on $E$. From Lemma 2, we define an operator $A: E \rightarrow E$ as follows:

$$
(A y)(t)=\int_{1}^{e} G(t, s) \varphi_{p}^{-1}\left(\int_{1}^{e} H(s, \tau) f(\tau, y(\tau)) \frac{d \tau}{\tau}\right) \frac{d s}{s}, y \in E .
$$

Note that the continuity of the functions $G, H, f$, guarantees that the operator $A$ is a completely continuous operator. Moreover if there is a $\bar{y} \in E$ such that $A \bar{y}=\bar{y}$, then from Lemma 2 we have that $\bar{y}$ is a solution for (1). Therefore, in what follows we study the existence of fixed points of the operator $A$.

Lemma 4. (see [32]). Let $E$ be a partially ordered Banach space, and $x_{0}, y_{0} \in E$ with $x_{0} \leq y_{0}, D=\left[x_{0}, y_{0}\right]$. Suppose that $A: D \rightarrow E$ satisfies the following conditions:

(i) $A$ is an increasing operator;

(ii) $x_{0} \leq A x_{0}, y_{0} \geq A y_{0}$, i.e., $x_{0}$ and $y_{0}$ is a subsolution and a supersolution of $A$; 
(iii) $A$ is a completely continuous operator.

Then $A$ has the smallest fixed point $x^{*}$ and the largest fixed point $y^{*}$ in $\left[x_{0}, y_{0}\right]$, respectively. Moreover, $x^{*}=\lim _{n \rightarrow \infty} A^{n} x_{0}$ and $y^{*}=\lim _{n \rightarrow \infty} A^{n} y_{0}$.

Lemma 5. (Avery-Henderson fixed point theorem, see [33]). Let $P$ be a cone in a real Banach space and for some positive constants $r$ and $\gamma$, there exist increasing, non-negative continuous functionals $\xi$ and $\zeta$ on $P$, and $a$ non-negative continuous functional $\vartheta$ on $P$ with $\vartheta(0)=0$, such that

$$
\zeta(y) \leq \vartheta(y) \leq \xi(y) \text {, and }\|y\| \leq \gamma \zeta(y), \forall y \in \overline{P(\zeta, r)} \text { with } P(\zeta, r)=\{y \in P: \zeta(y)<r\}
$$

Suppose that there exist positive numbers $\mu<v<r$ such that

$$
\vartheta(\lambda y) \leq \lambda \vartheta(y), \forall \lambda \in[0,1] \text { and } y \in \partial P(\vartheta, v)
$$

If $A: \overline{P(\zeta, r)} \rightarrow P$ is a completely continuous operator satisfying

(i) $\zeta(A y)>r$ for all $y \in \partial P(\zeta, r)$,

(ii) $\vartheta(A y)<v$ for all $y \in \partial P(\vartheta, v)$,

(iii) $P(\xi, \mu) \neq \varnothing$ and $\xi(A y)>\mu$ for all $y \in \partial P(\zeta, \mu)$,

Then $A$ has at least two fixed points $y_{1}$ and $y_{2}$ such that

$$
\mu<\xi\left(y_{1}\right) \text { with } \vartheta\left(y_{1}\right)<v ; v<\vartheta\left(y_{2}\right) \text { with } \zeta\left(y_{2}\right)<r
$$

Lemma 6. (see [23], Lemma 6). Let $\theta>0$ and $\psi \in P$. Then,

$$
\left(\int_{0}^{1} \psi(t) d t\right)^{\theta} \leq \int_{0}^{1} \psi^{\theta}(t) d t, \text { if } \theta \geq 1, \text { and }\left(\int_{0}^{1} \psi(t) d t\right)^{\theta} \geq \int_{0}^{1} \psi^{\theta}(t) d t \text {, if } 0<\theta \leq 1
$$

\section{Main Results}

In this section we let

$$
p_{*}=\min \{1, p-1\}, p^{*}=\max \{1, p-1\},
$$

and

$$
\omega(t)=(\log t)^{2}(1-\log t)^{\beta-2}, \sigma(t)=(\log t)^{\beta-2}(1-\log t)^{2}, t \in[1, e] .
$$

Now, we state our main results and give their proofs.

Theorem 1. Suppose that (H1)-(H2) and the following conditions hold:

(H3) There exist $a_{1} \geq\left(\frac{\Gamma(\beta)}{\beta-2}\right)^{p-1}\left[\int_{1}^{e} \int_{1}^{e} \omega^{p_{*}}(s) H^{\frac{p_{*}}{p-1}}(s, \tau) \sigma^{p_{*}}(\tau) \frac{d \tau}{\tau} \frac{d s}{s}\right]^{\frac{1-p}{p_{*}}}, r_{1}>0$ such that

$$
f(t, y) \geq a_{1} y^{p-1} \text { for } y \in\left[0, r_{1}\right], t \in[1, e] .
$$


(H4) There exist $a_{2} \in\left(0,2 \frac{p-p^{*}-1}{p^{*}}\left(\frac{\Gamma(\beta)}{M_{0}}\right)^{p-1}\left[\int_{1}^{e} \int_{1}^{e} H^{\frac{p^{*}}{p-1}}(s, \tau) \sigma^{p^{*}}(\tau) \frac{d \tau}{\tau} \frac{d s}{s}\right]^{\frac{1-p}{p^{*}}}\right), l_{1}>0$ such that

$$
f(t, y) \leq a_{2} y^{p-1}+l_{1} \text { for } y \in \mathbb{R}^{+}, t \in[1, e] .
$$

(H5) $f(t, y)$ is an increasing function in the second variable $y$, i.e., $f\left(t, y_{1}\right) \leq f\left(t, y_{2}\right)$ for $y_{1} \leq y_{2}, \forall t \in[1, e]$.

Then (1) has two positive solutions. Moreover, there exist two iterative sequences uniformly converging to the two solutions.

Proof. Using (H3) we have

$$
\begin{aligned}
{[(A y)(t)]^{p_{*}} } & =\left[\int_{1}^{e} G(t, s)\left(\int_{1}^{e} H(s, \tau) f(\tau, y(\tau)) \frac{d \tau}{\tau}\right) \frac{1}{p-1} \frac{d s}{s}\right]^{p_{*}} \\
& =\left[\int_{0}^{1} G\left(t, e^{s}\right)\left(\int_{0}^{1} H\left(e^{s}, e^{\tau}\right) f\left(e^{\tau}, y\left(e^{\tau}\right)\right) d \tau\right) \frac{1}{p-1} d s\right]^{p_{*}} \\
& \geq \int_{0}^{1} G^{p_{*}}\left(t, e^{s}\right)\left(\int_{0}^{1} H\left(e^{s}, e^{\tau}\right) f\left(e^{\tau}, y\left(e^{\tau}\right)\right) d \tau\right) \frac{p_{*}}{p-1} d s \\
& \geq \int_{0}^{1} G^{p_{*}}\left(t, e^{s}\right) \int_{0}^{1} \frac{p_{*}}{H^{p-1}}\left(e^{s}, e^{\tau}\right) \frac{p_{*}}{p-1}\left(e^{\tau}, y\left(e^{\tau}\right)\right) d \tau d s \\
& =\int_{1}^{e} G^{p_{*}}(t, s) \int_{1}^{e} H^{\frac{p_{*}}{p-1}}(s, \tau) f^{\frac{p_{*}}{p-1}}(\tau, y(\tau)) \frac{d \tau}{\tau} \frac{d s}{s} \\
& \geq \int_{1}^{e} G^{p_{*}}(t, s) \int_{1}^{e} H^{\frac{p_{*}}{p-1}}(s, \tau) a_{1}^{\frac{p_{*}}{p-1}} y^{p_{*}}(\tau) \frac{d \tau}{\tau} \frac{d s}{s} .
\end{aligned}
$$

Then, we can choose $\varepsilon_{1}>0$ such that $\varepsilon_{1} \sigma \in\left[0, r_{1}\right]$, and from Lemma 3 (iii) we have

$$
\begin{aligned}
{\left[\left(A \varepsilon_{1} \sigma\right)(t)\right]^{p_{*}} } & \geq \int_{1}^{e} G^{p_{*}}(t, s) \int_{1}^{e} \frac{p_{*}}{p-1}(s, \tau) a_{1}^{\frac{p_{*}}{p-1}} \varepsilon_{1}^{p_{*}} \sigma^{p_{*}}(\tau) \frac{d \tau}{\tau} \frac{d s}{s} \\
& \geq \int_{1}^{e}\left(\frac{\beta-2}{\Gamma(\beta)}\right)^{p_{*}} \omega^{p_{*}}(s) \sigma^{p_{*}}(t) \int_{1}^{e} H^{\frac{p_{*}}{p-1}}(s, \tau) a_{1}^{\frac{p_{*}}{p-1}} \varepsilon_{1}^{p_{*}} \sigma^{p_{*}}(\tau) \frac{d \tau}{\tau} \frac{d s}{s} \\
& \geq \varepsilon_{1}^{p_{*}} \sigma^{p_{*}}(t) .
\end{aligned}
$$

Thus

$$
A \varepsilon_{1} \sigma \geq \varepsilon_{1} \sigma
$$


On the other hand, from (H4) we have

$$
\begin{aligned}
{[(A y)(t)]^{p^{*}} } & =\left[\int_{1}^{e} G(t, s)\left(\int_{1}^{e} H(s, \tau) f(\tau, y(\tau)) \frac{d \tau}{\tau}\right) \overline{p-1} \frac{d s}{s}\right]^{p^{*}} \\
& \left.\left.=\left[\int_{0}^{1} G\left(t, e^{s}\right)\left(\int_{0}^{1} H\left(e^{s}, e^{\tau}\right) f\left(e^{\tau}, y\left(e^{\tau}\right)\right) d \tau\right)^{\frac{1}{p-1}} d s\right]\right]^{p^{*}}\right]^{\frac{p^{*}}{p-1}} d s \\
& \leq \int_{0}^{1} G^{p^{*}}\left(t, e^{s}\right)\left(\int_{0}^{1} H\left(e^{s}, e^{\tau}\right) f\left(e^{\tau}, y\left(e^{\tau}\right)\right) d \tau\right) \frac{p^{*}}{p^{*}} \\
& \leq \int_{0}^{1} G^{p_{*}}\left(t, e^{s}\right) \int_{0}^{1} H^{\frac{p}{p-1}}\left(e^{s}, e^{\tau}\right) f^{p-1}\left(e^{\tau}, y\left(e^{\tau}\right)\right) d \tau d s \\
& =\int_{1}^{e} G^{p^{*}}(t, s) \int_{1}^{e} H^{\frac{p^{*}}{p-1}}(s, \tau) f^{\frac{p^{*}}{p-1}}(\tau, y(\tau)) \frac{d \tau}{\tau} \frac{d s}{s} \\
& \leq \int_{1}^{e} G^{p^{*}}(t, s) \int_{1}^{e} H^{\frac{p^{*}}{p-1}}(s, \tau)\left(a_{2} y^{p-1}(\tau)+l_{1}\right) \frac{p^{*}}{p-1} \frac{d \tau}{\tau} \frac{d s}{s}
\end{aligned}
$$

Then from (H4) we have

$$
a_{2}^{\frac{p^{*}}{p-1}} 2^{\frac{p^{*}}{p-1}-1}\left(\frac{M_{0}}{\Gamma(\beta)}\right)^{p^{*}} \int_{1}^{e} \int_{1}^{e} H^{\frac{p^{*}}{p-1}}(s, \tau) \sigma^{p^{*}}(\tau) \frac{d \tau}{\tau} \frac{d s}{s}<1
$$

and hence there is a $M_{1}>\varepsilon_{1}$ and

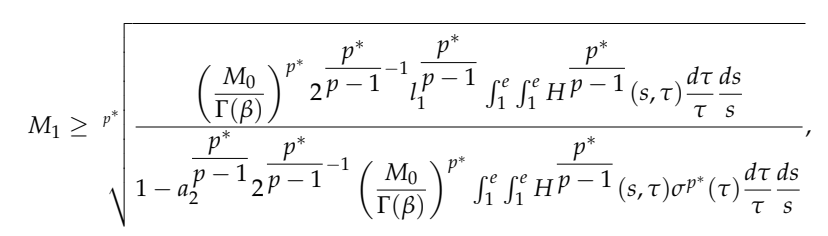

such that

$$
\begin{aligned}
{\left[\left(A M_{1} \sigma\right)(t)\right]^{p^{*}} \leq } & \int_{1}^{e} G^{p^{*}}(t, s) \int_{1}^{e} H^{\frac{p^{*}}{p-1}}(s, \tau)\left(a_{2} M_{1}^{p-1} \sigma^{p-1}(\tau)+l_{1}\right)^{\frac{p^{*}}{p-1}} \frac{d \tau}{\tau} \frac{d s}{s} \\
\leq & \int_{1}^{e} G^{p^{*}}(t, s) \int_{1}^{e} H^{\frac{p^{*}}{p-1}}(s, \tau) 2^{\frac{p^{*}}{p-1}}-1\left(\frac{p^{*}}{p-1} M_{1}^{p^{*}} \sigma^{p^{*}}(\tau)+l_{1}^{\frac{p^{*}}{p-1}}\right) \frac{d \tau}{\tau} \frac{d s}{s} \\
\leq & \int_{1}^{e}\left(\frac{M_{0}}{\Gamma(\beta)}\right)^{p^{*}} \sigma^{p^{*}}(t) \int_{1}^{e} H^{\frac{p^{*}}{p-1}}(s, \tau) 2^{\frac{p^{*}}{p-1}}-1\left(\frac{p^{*}}{a_{2}^{p-1}} M_{1}^{p^{*}} \sigma^{p^{*}}(\tau)+l_{1}^{\frac{p^{*}}{p-1}}\right) \frac{d \tau}{\tau} \frac{d s}{s} \\
= & M_{1}^{p^{*}} \sigma^{p^{*}}(t) \cdot a_{2}^{\frac{p^{*}}{p-1}} \frac{p^{*}}{p-1}-1 \\
& +\sigma^{p^{*}}(t) \cdot\left(\frac{M_{0}}{\Gamma(\beta)}\right)^{p^{*}} \int_{1}^{e} \int_{1}^{e} \frac{p^{*}}{\Gamma-1}(s, \tau) \sigma^{p^{*}}(\tau) \frac{d \tau}{\tau} \frac{d s}{s} \\
\leq & M_{1}^{p^{*}} \sigma^{p^{*}}(t) .
\end{aligned}
$$

This implies that

$$
A M_{1} \sigma \leq M_{1} \sigma
$$


Iin Lemma 4 we put $D=\left[\varepsilon_{1} \sigma, M_{1} \sigma\right]$, and from (H5) we have that $A$ is an increasing operator. Thus from Lemma $4, A$ has the smallest fixed point $y_{* *}$ and the largest fixed point $y^{* *}$ in $D$. That is, Equation (1) has two positive solutions $y_{* *}$ and $y^{* *}$ in $D$. Moreover, $y_{* *}=\lim _{n \rightarrow \infty} A^{n} \varepsilon_{1} \sigma=$ $\lim _{n \rightarrow \infty} A^{n-1}\left(A \varepsilon_{1} \sigma\right)$ and $y^{* *}=\lim _{n \rightarrow \infty} A^{n} M_{1} \sigma=\lim _{n \rightarrow \infty} A^{n-1}\left(A M_{1} \sigma\right)$. This completes the proof.

Lemma 7. Let $P_{0}=\left\{y \in P: y(t) \geq \frac{(\beta-2) \kappa^{*}}{M_{0}}\|y\|, t \in\left[\kappa_{1}, \kappa_{2}\right]\right\}$. Then $A(P) \subset P_{0}$, where $\kappa_{1}, \kappa_{2} \in(1, e)$ with $\kappa_{1}<\kappa_{2}$, and $\kappa^{*}=\min _{t \in\left[\kappa_{1}, \kappa_{2}\right]} \sigma(t)>0$.

Proof. For $y \in P$, from Lemm 3 (ii) we have

$$
\begin{aligned}
(A y)(t) & =\int_{1}^{e} G(t, s) \varphi_{p}^{-1}\left(\int_{1}^{e} H(s, \tau) f(\tau, y(\tau)) \frac{d \tau}{\tau}\right) \frac{d s}{s} \\
& \leq \frac{M_{0}}{\Gamma(\beta)} \int_{1}^{e} \omega(s) \varphi_{p}^{-1}\left(\int_{1}^{e} H(s, \tau) f(\tau, y(\tau)) \frac{d \tau}{\tau}\right) \frac{d s}{s}
\end{aligned}
$$

and

$$
\begin{aligned}
(A y)(t) & =\int_{1}^{e} G(t, s) \varphi_{p}^{-1}\left(\int_{1}^{e} H(s, \tau) f(\tau, y(\tau)) \frac{d \tau}{\tau}\right) \frac{d s}{s} \\
& \geq \frac{\beta-2}{\Gamma(\beta)} \int_{1}^{e} \sigma(t) \omega(s) \varphi_{p}^{-1}\left(\int_{1}^{e} H(s, \tau) f(\tau, y(\tau)) \frac{d \tau}{\tau}\right) \frac{d s}{s} \\
& =\frac{\beta-2}{M_{0}} \sigma(t) \cdot \frac{M_{0}}{\Gamma(\beta)} \int_{1}^{e} \omega(s) \varphi_{p}^{-1}\left(\int_{1}^{e} H(s, \tau) f(\tau, y(\tau)) \frac{d \tau}{\tau}\right) \frac{d s}{s} .
\end{aligned}
$$

Therefore, we have

$$
(A y)(t) \geq \frac{\beta-2}{M_{0}} \sigma(t)\|A y\|, t \in[1, e] .
$$

In particular, we have

$$
(A y)(t) \geq \frac{(\beta-2) \kappa^{*}}{M_{0}}\|A y\|, t \in\left[\kappa_{1}, \kappa_{2}\right] .
$$

This completes the proof.

Let

$$
I=\left[\kappa_{1}, \kappa_{2}\right], \zeta(y)=\min _{t \in I} y(t), \vartheta(y)=\max _{t \in I} y(t), \xi(y)=\max _{t \in[1, e]} y(t) \text {, and } P(\zeta, r)=\left\{y \in P_{0}: \zeta(y)<r\right\},
$$

and

$$
C=\frac{(\beta-2) \kappa^{*}}{\Gamma(\beta)} \int_{1}^{e} \omega(s) \varphi_{p}^{-1}\left(\int_{I} H(s, \tau) \frac{d \tau}{\tau}\right) \frac{d s}{s}, D=\frac{M_{0}}{\Gamma(\beta)} \int_{1}^{e} \omega(s) \varphi_{p}^{-1}\left(\int_{1}^{e} H(s, \tau) \frac{d \tau}{\tau}\right) \frac{d s}{s} .
$$

Theorem 2. Suppose that (H1)-(H2) hold, and there exist positive constants $0<\mu<v<r$ such that the function $f$ satisfies the following conditions:

(H6) $f(t, y)>\varphi_{p}\left(\frac{\mu}{C}\right)$ for $t \in I$ and $y \in\left[\frac{(\beta-2) \kappa^{*}}{M_{0}} \mu, \mu\right]$,

(H7) $f(t, y)<\varphi_{p}\left(\frac{v}{D}\right)$ for $t \in[1, e]$ and $y \in\left[0, \frac{v M_{0}}{(\beta-2) \kappa^{*}}\right]$,

(H8) $f(t, y)>\varphi_{p}\left(\frac{r}{C}\right)$ for $t \in I$ and $y \in\left[r, \frac{r M_{0}}{(\beta-2) \kappa^{*}}\right]$. 
Then (1) has at least two positive solutions $y_{1}$ and $y_{2}$ such that

$$
\begin{aligned}
& \mu<\max _{t \in[1, e]} y_{1}(t) \text { with } \max _{t \in I} y_{1}(t)<v, \\
& v<\max _{t \in I} y_{2}(t) \text { with } \min _{t \in I} y_{2}(t)<r .
\end{aligned}
$$

Proof. Note that $P_{0} \subset P$, and from Lemma 7 we have $A\left(P_{0}\right) \subset P_{0}$. From the definitions of $\zeta, \xi, \vartheta$, for each $y \in P_{0}$ we have

$$
\zeta(y) \leq \vartheta(y) \leq \xi(y), \text { and }\|y\| \leq \frac{M_{0}}{(\beta-2) \kappa^{*}} \min _{t \in I} y(t)=\frac{M_{0}}{(\beta-2) \kappa^{*}} \zeta(y) .
$$

For every $y \in P_{0}, \lambda \in[0,1]$ we obtain

$$
\vartheta(\lambda y)=\max _{t \in I} \lambda y(t)=\lambda \vartheta(y), \text { and } \vartheta(0)=0
$$

We first verify condition (iii) in Lemma 5. Since $0 \in P_{0}$ and $\mu>0, P(\xi, \mu) \neq \varnothing$. Note from $y \in \partial P(\xi, \mu)$,i.e., $\|y\|=\mu$, and thus $\frac{(\beta-2) \kappa^{*}}{M_{0}} \mu \leq y(t) \leq \mu$ for $t \in I$. Therefore, using (H6) we have

$$
\begin{aligned}
\xi(A y) & =\max _{t \in[1, e]}(A y)(t) \\
& =\max _{t \in[1, e]} \int_{1}^{e} G(t, s) \varphi_{p}^{-1}\left(\int_{1}^{e} H(s, \tau) f(\tau, y(\tau)) \frac{d \tau}{\tau}\right) \frac{d s}{s} \\
& \geq \max _{t \in[1, e]} \frac{\beta-2}{\Gamma(\beta)} \int_{1}^{e} \sigma(t) \omega(s) \varphi_{p}^{-1}\left(\int_{1}^{e} H(s, \tau) f(\tau, y(\tau)) \frac{d \tau}{\tau}\right) \frac{d s}{s} \\
& \geq \max _{t \in I} \frac{\beta-2}{\Gamma(\beta)} \int_{1}^{e} \sigma(t) \omega(s) \varphi_{p}^{-1}\left(\int_{1}^{e} H(s, \tau) f(\tau, y(\tau)) \frac{d \tau}{\tau}\right) \frac{d s}{s} \\
& \geq \min _{t \in I} \frac{\beta-2}{\Gamma(\beta)} \int_{1}^{e} \sigma(t) \omega(s) \varphi_{p}^{-1}\left(\int_{1}^{e} H(s, \tau) f(\tau, y(\tau)) \frac{d \tau}{\tau}\right) \frac{d s}{s} \\
& >\frac{(\beta-2) \kappa^{*}}{\Gamma(\beta)} \int_{1}^{e} \omega(s) \varphi_{p}^{-1}\left(\int_{I} H(s, \tau) \varphi_{p}\left(\frac{\mu}{C}\right) \frac{d \tau}{\tau}\right) \frac{d s}{s} \\
& =\frac{\mu}{C} \frac{(\beta-2) \kappa^{*}}{\Gamma(\beta)} \int_{1}^{e} \omega(s) \varphi_{p}^{-1}\left(\int_{I} H(s, \tau) \frac{d \tau}{\tau}\right) \frac{d s}{s} \\
& =\mu .
\end{aligned}
$$

We next show condition (ii) in Lemma 5 is true. Since $y \in \partial P(\vartheta, v)$, then $0 \leq y(t) \leq\|y\| \leq$ $\frac{v M_{0}}{(\beta-2) \kappa^{*}}$ for $t \in[1, e]$. Consequently, from (H7) we obtain

$$
\begin{aligned}
\vartheta(A y) & =\max _{t \in I}(A y)(t) \\
& =\max _{t \in I} \int_{1}^{e} G(t, s) \varphi_{p}^{-1}\left(\int_{1}^{e} H(s, \tau) f(\tau, y(\tau)) \frac{d \tau}{\tau}\right) \frac{d s}{s} \\
& \leq \frac{M_{0}}{\Gamma(\beta)} \int_{1}^{e} \omega(s) \varphi_{p}^{-1}\left(\int_{1}^{e} H(s, \tau) f(\tau, y(\tau)) \frac{d \tau}{\tau}\right) \frac{d s}{s} \\
& <\frac{M_{0}}{\Gamma(\beta)} \int_{1}^{e} \omega(s) \varphi_{p}^{-1}\left(\int_{1}^{e} H(s, \tau) \varphi_{p}\left(\frac{v}{D}\right) \frac{d \tau}{\tau}\right) \frac{d s}{s} \\
& =\frac{v}{D} \frac{M_{0}}{\Gamma(\beta)} \int_{1}^{e} \omega(s) \varphi_{p}^{-1}\left(\int_{1}^{e} H(s, \tau) \frac{d \tau}{\tau}\right) \frac{d s}{s} \\
& =v .
\end{aligned}
$$


Finally, we show condition (i) in that Lemma 5 holds. Since $y \in \partial P(\zeta, r)$, i.e., $\min _{t \in I} y(t)=r$, and thus $r \leq y(t) \leq\|y\| \leq \frac{r M_{0}}{(\beta-2) \kappa^{*}}$ for $t \in I$. Hence, (H8) implies that

$$
\begin{aligned}
\zeta(A y) & =\min _{t \in I}(A y)(t) \\
& =\min _{t \in I} \int_{1}^{e} G(t, s) \varphi_{p}^{-1}\left(\int_{1}^{e} H(s, \tau) f(\tau, y(\tau)) \frac{d \tau}{\tau}\right) \frac{d s}{s} \\
& \geq \min _{t \in I} \frac{\beta-2}{\Gamma(\beta)} \int_{1}^{e} \sigma(t) \omega(s) \varphi_{p}^{-1}\left(\int_{1}^{e} H(s, \tau) f(\tau, y(\tau)) \frac{d \tau}{\tau}\right) \frac{d s}{s} \\
& >\frac{(\beta-2) \kappa^{*}}{\Gamma(\beta)} \int_{1}^{e} \omega(s) \varphi_{p}^{-1}\left(\int_{I} H(s, \tau) \varphi_{p}\left(\frac{r}{C}\right) \frac{d \tau}{\tau}\right) \frac{d s}{s} \\
& =\frac{r}{C} \frac{(\beta-2) \kappa^{*}}{\Gamma(\beta)} \int_{1}^{e} \omega(s) \varphi_{p}^{-1}\left(\int_{I} H(s, \tau) \frac{d \tau}{\tau}\right) \frac{d s}{s} \\
& =r .
\end{aligned}
$$

This completes the proof.

\section{Conclusions}

In this paper, we first used the monotone iterative technique to show that (1) has two positive solutions, and we established iterative formulas for the two solutions when the nonlinearity $f$ grows $(p-1)$-sublinearly. Next, using the Avery-Henderson fixed point theorem, we showed that (1) has two positive solutions under some appropriate conditions on the nonlinearity $f$.

Author Contributions: Conceptualization, J.J., J.X. and D.O..; methodology, J.J., J.X. and D.O.; software, J.J.; validation, J.J., J.X. and D.O.; formal analysis, J.J., J.X. and D.O.; investigation, J.J., J.X. and D.O.; resources, J.X.; data curation, J.X.; writing original draft preparation, J.J., J.X. and D.O.; writing review and editing, J.J., J.X. and D.O..; visualization, J.X.; supervision, J.X. and D.O.; project administration, J.X.; funding acquisition, J.X. All authors have read and agreed to the published version of the manuscript.

Funding: This work is supported by Talent Project of Chongqing Normal University (02030307-0040), China Postdoctoral Science Foundation (Grant No. 2019M652348), Technology Research Foundation of Chongqing Educational Committee(Grant No. KJQN201900539), Natural Science Foundation of Chongqing Normal University (Grant No. 16XYY24).

Conflicts of Interest: The authors declare no conflict of interest.

\section{References}

1. Podlubny, I. Fractional Differential Equations. In Mathematics in Science and Engineering; Academic Press: San Diego, CA, USA, 1999; Volume 198.

2. Kilbas, A.A.; Srivastava, H.M.; Trujillo, J.J. Theory and Applications of Fractional Differential Equations. In North-Holland Mathematics Studies; Elsevier: Amsterdam, The Netherlands, 2006; Volume 204.

3. Samko, S.G.; Kilbas, A.A.; Marichev, O.I. Fractional Integrals and Derivatives, Theory and Applications; Gordon and Breach: Yverdon, Switzerland, 1993.

4. Diethelm, K. The Analysis of Fractional Differential Equations: An Application-Oriented Exposition Using Differential Operators of Caputo Type; Springer: Heidelberg, Germany, 2010.

5. Ahmad, B.; Alsaedi, A.; Ntouyas, S.K.; Tariboon, J. Hadamard-Type Fractional Differential Equations, Inclusions and Inequalities; Springer: New York City, NY, USA, 2017.

6. Diethelm, K.; Ford, N.J. Multi-order fractional differential equations and their numerical solution. Appl. Math. Comput. 2004, 154, 621-640. [CrossRef]

7. Luchko, Y. Some uniqueness and existence results for the initial-boundary-value problems for the generalized time-fractional diffusion equation. Comput. Math. Appl. 2010, 59, 1766-1772. [CrossRef]

8. Luchko, Y. Fractional wave equation and damped waves. J. Math. Phys. 2013, 54, 031505. [CrossRef] 
9. Luchko, Y. Boundary value problems for the generalized time-fractional diffusion equation of distributed order. Fract. Calc. Appl. Anal. 2009, 12, 409-422.

10. Machado, J.T.; Kiryakova, V.; Mainardi, F. Recent history of fractional calculus. Commun. Nonlinear Sci. Numer. Simul. 2011, 16, 1140-1153. [CrossRef]

11. Kilbas, A.A.; Bonilla, B.; Trujillo, J.J. Existence and uniqueness theorems for nonlinear fractional differential equations. Demonstratio Math. 2000, 33, 583-602. [CrossRef]

12. Kilbas, A.A.; Trujillo, J.J. Differential equations of fractional order: methods, results and problems-I. Appl. Anal. 2001, 78, 153-192. [CrossRef]

13. Kilbas, A.A.; Trujillo, J.J. Differential equations of fractional order: methods, results and problems-II. Appl. Anal. 2002, 81, 435-493. [CrossRef]

14. Xu, X.; Jiang, D.; Yuan, C. Multiple positive solutions for the boundary value problem of a nonlinear fractional differential equation. Nonlinear Anal. 2009, 71, 4676-4688. [CrossRef]

15. Hao, X.; Wang, H.; Liu, L.; Cui, Y. Positive solutions for a system of nonlinear fractional nonlocal boundary value problems with parameters and p-Laplacian operator. Bound. Value Probl. 2017, 2017, 182. [CrossRef]

16. Dong, X.; Bai, Z.; Zhang, S. Positive solutions to boundary value problems of $p$-Laplacian with fractional derivative. Bound. Value Probl. 2017, 2017, 5. [CrossRef]

17. Wu, J.; Zhang, X.; Liu, L.; Wu, Y.; Cui, Y. The convergence analysis and error estimation for unique solution of a $p$-Laplacian fractional differential equation with singular decreasing nonlinearity. Bound. Value Probl. 2018, 2018, 82. [CrossRef]

18. Cheng, W.; Xu, J.; O’Regan, D.; Cui, Y. Positive solutions for a nonlinear discrete fractional boundary value problem with a $p$-Laplacian operator. J. Anal. Appl. Comput. 2019, 9, 1959-1972.

19. Ahmad, B.; Ntouyas, S.K. On Hadamard fractional integro-differential boundary value problems. J. Appl. Math. Comput. 2015, 47, 119-131. [CrossRef]

20. Wang, T; Wang, G.; Yang, X. On a Hadamard-type fractional turbulent flow model with deviating arguments in a porous medium. Nonlinear Anal. Model. Control 2017, 22, 765-784. [CrossRef]

21. Wang, G.; Ren, X.; Zhang, L.; Ahmad, B. Explicit iteration and unique positive solution for a Caputo-Hadamard fractional turbulent flow model. IEEE Access 2019, 7, 109833-109839. [CrossRef]

22. Zhang, K.; Wang, J.; Ma, W. Solutions for integral boundary value problems of nonlinear Hadamard fractional differential equations. J. Funct. Spaces 2018, 2018, 2193234. [CrossRef]

23. Jiang, J.; O’Regan, D.; Xu, J.; Cui, Y. Positive solutions for a Hadamard fractional $p$-Laplacian three-point boundary value problem. Mathematics 2019, 7, 439. [CrossRef]

24. Zhang, H.; Li, Y.; Xu, J. Positive solutions for a system of fractional integral boundary value problems involving Hadamard-type fractional derivatives. Complexity 2019, 2019, 2671539. [CrossRef]

25. Ding, Y.; Jiang, J.; O’Regan, D.; Xu, J. Positive solutions for a system of Hadamard-type fractional differential equations with semipositone nonlinearities. Complexity 2020, 2020, 9742418. [CrossRef]

26. Jiang, J.; O'Regan, D.; Xu, J.; Fu, Z. Positive solutions for a system of nonlinear Hadamard fractional differential equations involving coupled integral boundary conditions. J. Inequal. Appl. 2019, 2019, 204. [CrossRef]

27. Zhang, K.; Fu, Z. Solutions for a class of Hadamard fractional boundary value problems with sign-changing nonlinearity. J. Funct. Spaces 2019, 2019, 9046472. [CrossRef]

28. Zhai, C.; Wang, W.; Li, H. A uniqueness method to a new Hadamard fractional differential system with four-point boundary conditions. J. Inequal. Appl. 2018, 2018, 207. [CrossRef] [PubMed]

29. Riaz, U.; Zada, A.; Ali, Z.; Ahmad, M.; Xu, J.; Fu, Z. Analysis of nonlinear coupled systems of impulsive fractional differential equations with Hadamard derivatives. Math. Probl. Eng. 2019, 2019, 5093572. [CrossRef]

30. Riaz, U.; Zada, A.; Ali, Z.; Cui, Y.; Xu, J. Analysis of coupled systems of implicit impulsive fractional differential equations involving Hadamard derivatives. Adv. Differ. Equ. 2019, 2019, 226. [CrossRef]

31. Leibenson, L.S. General problem of the movement of a compressible fluid in porous medium. Izv. Akad. Nauk SSSR 1945, 9, 7-10. 
32. Guo, D.; Lakshmikantham, V. Nonlinear Problems in Abstract Cones; Academic Press: Orlando, FL, USA, 1988.

33. Avery, R.I.; Henderson, J. Two positive fixed points of nonlinear operators on ordered Banach spaces. Commun. Appl. Nonlinear Anal. 2001, 8, 27-36.

(C) 2020 by the authors. Licensee MDPI, Basel, Switzerland. This article is an open access article distributed under the terms and conditions of the Creative Commons Attribution (CC BY) license (http://creativecommons.org/licenses/by/4.0/). 